\title{
The sexuality of Phlebiopsis gigantea
}

\author{
KARI KORHONEN and PIRKKO KAUPPILA
}

KORHONEN, K. \& KAUPPILA, P. 1988: The sexuality of Phlebiopsis gigantea. Karstenia 27: 23-30.

The mating system of Phlebiopsis gigantea (Fr.) Jülich, a common decay fungus of coniferous wood and a biological control agent used against Heterobasidion annosum (Fr.) Bref., is shown to be bipolar. Clamp connections can be found in both hetero- and homokaryotic mycelia. Homokaryotic fruiting occurs fairly frequently in pure cultures. Collections from Finland, Poland and France proved to be interfertile.

Kari Korhonen, The Finnish Forest Research Institute, P.O. Box 18, SF-01301 Vantaa, Finland

Pirkko Kauppila, Muskettitie 12, SF-02680 Espoo, Finland

Phlebiopsis gigantea (Fr.) Jülich (Basidiomycetes, Corticiaceae; syn. Phlebia gigantea (Fr.) Donk, Peniophora gigantea (Fr.) Massee) is a common saprophytic fungus decaying coniferous wood, especially pine (Käärik \& Rennerfelt 1957). It is an effective rotting agent, and one of the most important destroyers of stored timber. Since it is a strong competitor of the serious root pathogen Heterobasidion annosum (Fr.) Bref. in pine stumps, $P$. gigantea has been used as a biological control agent to prevent the penetration of $H$. annosum through the cut surface of such stumps (Rishbeth 1979).

$P$. gigantea is an easily recognized species, being clearly distinguished from its relatives (Eriksson et al. 1981). The resupinate fruit bodies are grey, somewhat translucent when moist, and have characteristic conical cystidia projecting from the hymenium. Pure cultures of $P$. gigantea can usually be identified without difficulty on the basis of their oidial chains, encrusted upright aerial hyphae, and multiple clamp connections (Stalpers 1978).

Biggs (1938) and Boidin (1950) reported that the cells in the hyphae of $P$. gigantea are multinucleate. Binucleate cells can be found in the subhymenium, and the cystidia and spores are generally also binucleate. Multiple clamp connections (two or more at the same septum) are present in both single-spore and tissue isolates.

Besides $P$. gigantea, there are a number of other species within the Hymenomycetes which develop clamp connections in single-basidiospore cultures. This property has generally been considered an indication of homothallism, either primary or secondary. It often occurs together with holocoenocytic nuclear behaviour (multinucleate cells in primary and secondary mycelia). Of course, it is not a sure proof of homothallism, and the existence of clamp connections in homokaryotic mycelia of a heterothallic species has recently been demonstrated in a species of Coprinus (Kemp 1980), and in some species of Stereum (Coates et al. 1981, Boddy \& Rayner 1982, Rayner \& Turton 1982).

In their review on the sexuality of basidiomycetes, Boidin and Lanquetin (1984a, b) used the term "presumed homothallism" when dealing with species showing holocoenocytic nuclear behaviour and clamp connections in single-spore mycelia. $P$. gigantea is listed as one of these presumably homothallic species. Here we report the results of mating experiments which show that $P$. gigantea is a heterothallic fungus in which clamp connections may occur in homokaryotic mycelia. 


\section{Material and methods}

Seventy fruit bodies of $P$. gigantea were collected from several places in southern Finland and one specimen from France (Eure). Thirty-nine specimens originated from Norway spruce (Picea abies (L.) Karsten), 30 from Scots pine (Pinus sylvestris L.) and one from lodgepole pine (Pinus contorta Douglas ex Loudon). Ten or twelve single-basidiospore cultures and a tissue culture were isolated from each fruit body (Korhonen \& Hintikka 1981) and stored in test tubes on malt extract agar slants at $2-4^{\circ} \mathrm{C}$.

Pairings were made on malt extract agar (1\% ME) in Petri dishes. Although several other culture media and various culture conditions were tested in order to enhance the mating between single-spore mycelia, the standard medium finally proved to be the best. Fruiting of the cultures was induced by incubating the Petri dishes at room temperature in diffuse daylight for about three months, until the culture medium dried out.

A search for clamp connections was made on young colonies that had been grown in Petri dishes for about one week. Two- or three-month-old cultures were inspected for fruit bodies. The first sign of a developing fruit body was the appearance of cystidia (Fig. 1) on the surface of the agar medium, often along the margins of the dish. Mature fruit bodies were generally easy to detect because the basidiospores shoot out to a distance of several millimetres: the spore print can be seen on the lid of the Petri dish when held in the normal upright position.

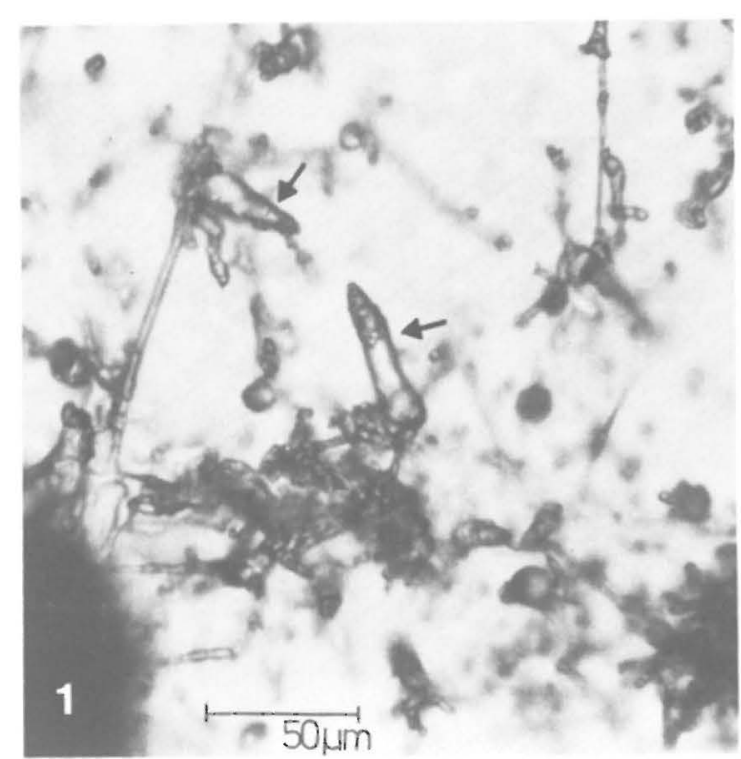

Fig. 1. Cystidia of a developing fruit body on the surface of agar medium.

\section{Results}

Most of the septa in pure cultures of $P$. gigantea are simple. The clamp connections, which may be single or multiple, seem to occur quite irregularly along the hyphae.

Tissue isolates and single-basidiospore isolates resemble each other in many respects. Both have multinucleate cells, both may have clamp connections, and both may fruit in pure culture. However, a comparison of these mycelial types also revealed slight morphological differences and clear signs of heterokaryosis in tissue isolates and homokaryosis in single-spore isolates.

Heterokaryotic cultures. The surface of a heterokaryotic culture (Fig. 2) is usually thinly covered by short aerial hyphae with crystalline encrustments and by oidial chains. The clamp connections, many of them multiple, are usually numerous but vary in number. At least some clamp connections appear to be present in all heterokaryotic cultures. The heterokaryons grow quickly (about $7 \mathrm{~mm} /$ day at $20^{\circ} \mathrm{C}$ ), in a regular manner, and fruit readily after two to three months.

Homokaryotic cultures. Homokaryotic cultures (Fig. 2) have generally more aerial mycelium, somewhat thinner hyphae and a slower growth rate (about $5 \mathrm{~mm} / \mathrm{day}$ ) than the tissue cultures. The number of oidia is usually high, but encrusted hyphae are less numerous or completely lacking. The morphological differences between single-spore and tissue cultures are not always clear-cut.

The occurrence of clamp connections was checked in 280 single-spore isolates, derived from 40 natural fruit bodies, seven isolates from each. Multiple clamp connections were found in 107 isolates (38.2\%), single ones in $63(22.5 \%)$, and none in 110 isolates (39.3\%). Most fruit bodies produced both clamped and clampless single-spore mycelia. Some of the clamped single-spore cultures were heterokaryotic but most of them were homokaryotic, judging by their behaviour in matings.

Hetero- and homokaryotic fruiting. The single-basidiospore cultures isolated from natural fruit bodies and those derived from fruiting heterokaryotic cultures were somewhat different in external appearance. When paired, they often formed a more or less distinct line of demarcation between each other, indicating genetic differences (Fig. 3). These differences result from the meiotic segregation in the heterokaryotic fruit body.

Most single-spore cultures did not fruit in Petri dishes within three months. Some fruited (about $22 \%$ ), but in most cases the single-spore progeny of these fruit bodies proved to be genetically identical (Fig. 3). The homokaryotic fruit bodies were usually 

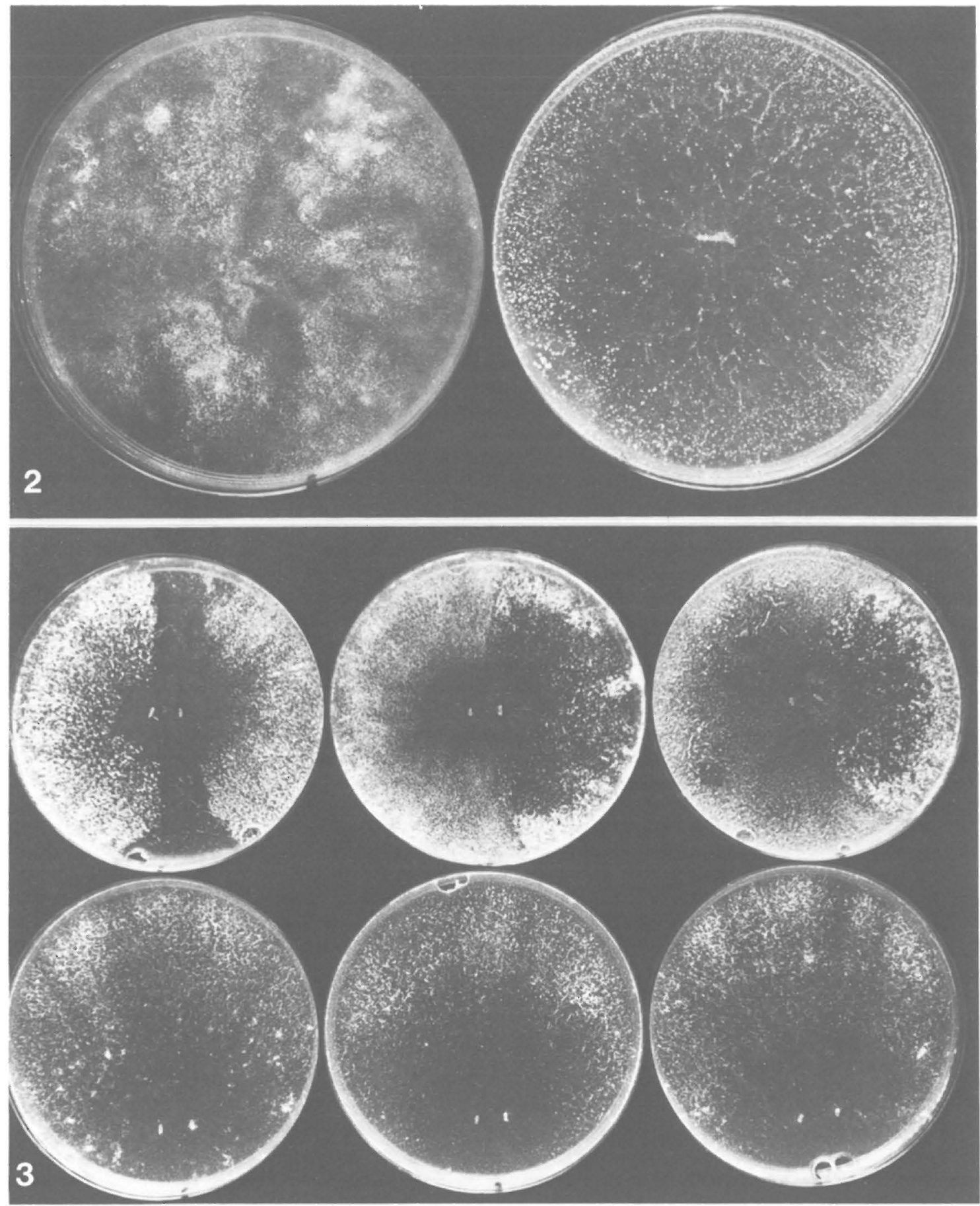

Figs. 2-3. Phlebiopsis gigantea. - 2: Typical appearance of pure homokaryotic (left) and heterokaryotic (right) cultures. Age of cultures four weeks. - 3: Pairings between single-basidiospore mycelia isolated from a heterokaryotic (upper row) and a homokaryotic (lower row) fruit body. The isolates from the heterokaryotic fruit body differ from each other, while the isolates from the homokaryotic fruit body are all identical. Age of cultures four weeks. 
smaller and produced fewer spores than the heterokaryotic ones. All the 70 fruit bodies collected in the field were heterokaryotic.

Nuclear condition in oidia. Single-oidium cultures isolated from a heterokaryotic mycelium could usually be divided into three different types: one heterokaryotic (like the original) and two homokaryotic types. When the two latter types were mated with each other, the original heterokaryon was again synthesized. All the single-oidium mycelia isolated from a homokaryotic culture were identical with the original culture.

Mating system. Although heterothallism in $P$. gigantea was clearly demonstrated in the experiments described above, it proved rather difficult to achieve a final solution of the mating system. Our first mating experiments were made with cultures which had been isolated one or two years earlier. The results were frustrating: we were unable to find any regular mating pattern despite several attempts on different culture media.

This failure was partially due to rapid senescence of the cultures. On closer examination it became evident that many of the homokaryotic cultures had apparently lost their ability to mate after storage for one year at $2-4^{\circ} \mathrm{C}$.

Fresh material, consisting of 40 fruit bodies (20 from Norway spruce, 20 from Scots pine), was collected, and 10-12 single-spore cultures isolated from each. Soon after isolation, 7-10 single-spore mycelia from each fruit body (a stock) were paired with each other in all combinations. These pairings were incubated in daylight in the laboratory, and examined after two and three months. A mating was regarded as compatible if basidia or cystidia were present. On the basis of these criteria a perfect bipolar mating pattern was found in eight of the stocks. The mating pattern of the remaining 32 stocks was also interpreted as being bipolar, although more or less irregular (Fig. 4). Occasional fruiting of homokaryotic mycelia and occasional sterility of heterokaryotic mycelia apparently explain these irregularities.

In compatible matings, the external mating reactions were variable. It was usually impossible to distinguish between compatible and incompatible matings after three or four weeks. Sometimes a heterokaryotic mycelium started to grow from the contact area between homokaryons soon after the mycelia had met each other, but in most cases the hetero-

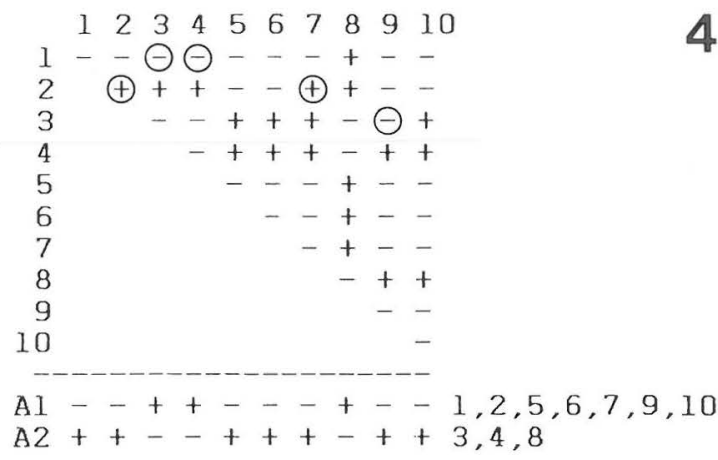

Fig. 4. A typical result obtained when 10 single-spore isolates from one fruit body were paired with each other. + indicates fruiting, - no fruiting, circled markes are exceptions to the bipolar mating pattern due to homokaryotic fruiting $(+)$ or lack of heterokaryotic fruiting (-).

karyotic mycelium appeared to have been formed at some later stage (Fig. 5). We did not observe any septal breakdown or other signs of nuclear migration. In incompatible pairings the line of demarcation between the homokaryotic partners was often unclear (Fig. 6).

Interfertility. The interfertility between different stocks was first investigated by pairing two compatible homokaryons from 10 stocks in all combinations. These stocks originated from different parts of South Finland. All of them proved to be interfertile. At least 17 different alleles of the incompatibility factor were found among 20 possible. The exact number could not be determined reliably owing to certain anomalies in the mating pattern.

Four homokaryotic tester strains were selected from the previous experiment and paired with two homokaryotic non-fruiting isolates from all the remaining stocks (including the older collections). In addition to the stocks from Finland and France, homokaryotic single-oidium isolates from three Polish isolates were tested (kindly supplied by Dr. Z. Sierota, Warsaw). All these stocks proved to be interfertile with the testers, although there were some minor irregularities among the matings, probably due to the senescence of some cultures.

Buller phenomenon. When homokaryotic tester strains were paired with heterokaryotic cultures, the testers often slowly turned heterokaryotic

Figs. 5-6. Phlebiopsis gigantea. - 5: Two compatible matings at the age of three weeks. In mating (b) the heterokaryotic mycelium has arisen soon after the homokaryotic mycelia have met each other. In mating (a), which is more usual, the formation of heterokaryotic mycelium is not as apparent. - 6: A compatible and an incompatible mating at the age of two months. In the incompatible mating $(1 \times 3)$ the homokaryotic partners remain separate. In the compatible mating ( $7 \times 14)$ the heterokaryon has overgrown the homokaryons, and fruit bodies are developing along the margin of the dish (arrows). 


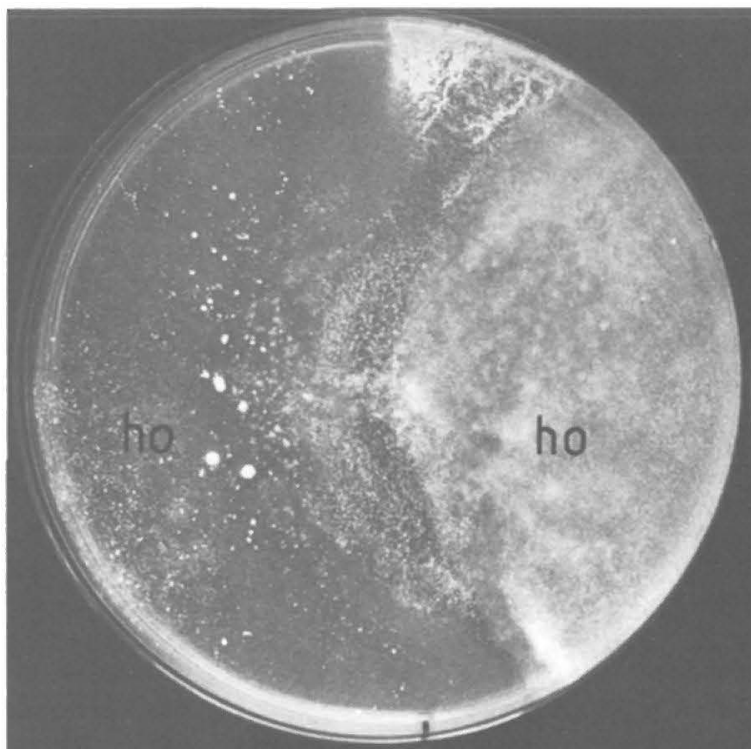

5

a

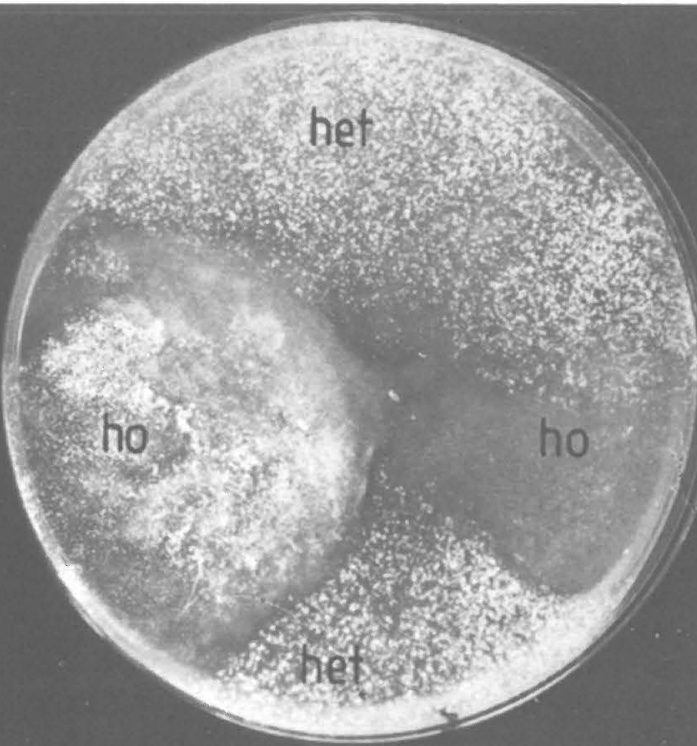

b

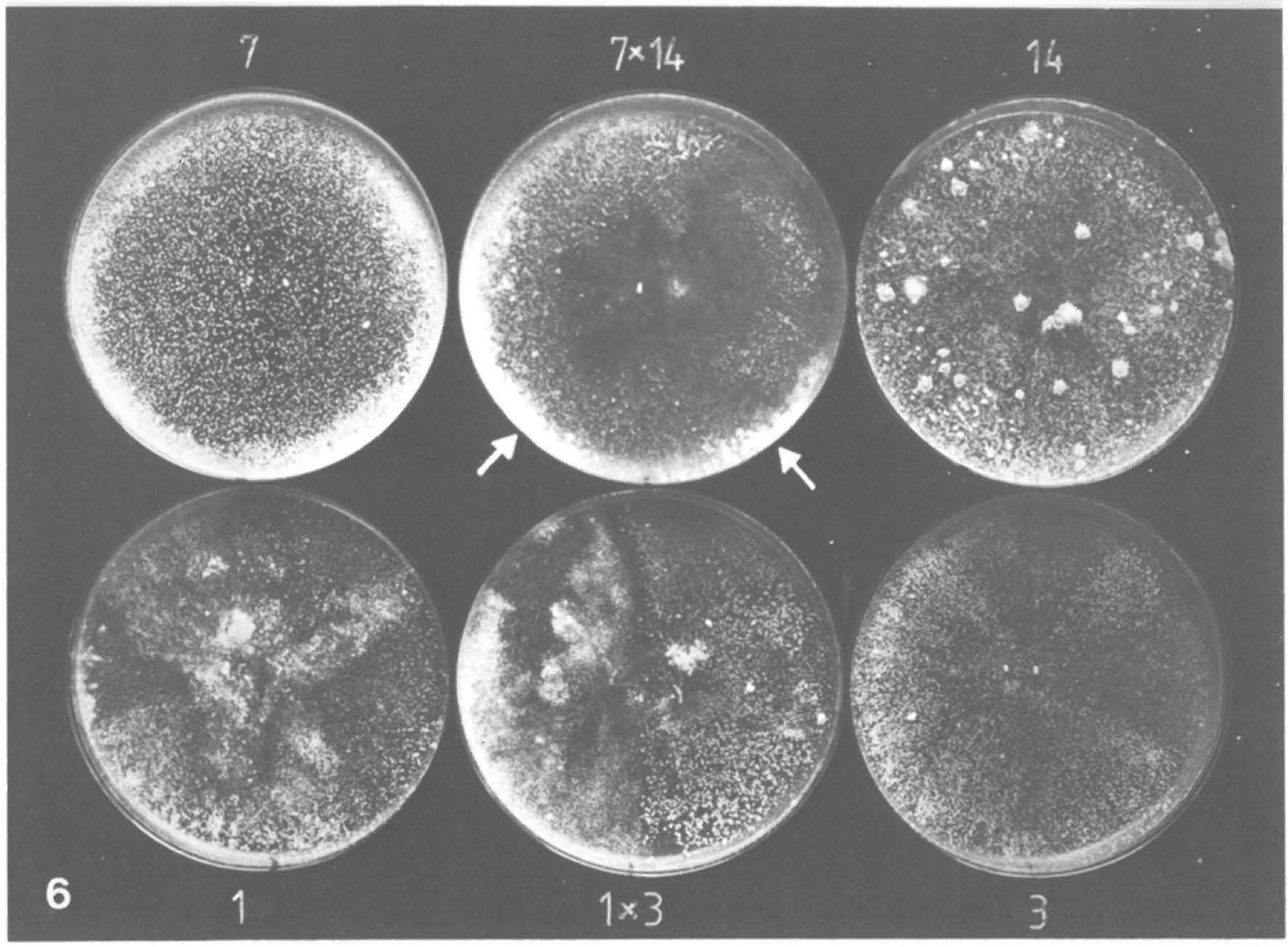


as a result of the Buller phenomenon or overgrowth by the heterokaryon. However, the functioning of these phenomena in pairings of $P$. gigantea was irregular, and in many pairings the tester side of the dish retained its homokaryotic appearance and did not fruit within three months.

\section{Discussion}

Although multiple clamp connections can be found in many species of the non-poroid Aphyllophorales (Boidin \& Lanquetin 1984b), they are very rare among polypores and agarics (Stalpers 1978, SchulzWeddingen 1985, Gilbertson \& Ryvarden 1986). The significance of multiple clamp connections is poorly understood. So also is the significance of occasional clamp connections, single or multiple, on hyphae of those species which mostly have simple septa. In actual fact, the overall importance of the clamp connection for many basidiomycetes is somewhat enigmatic.

In a tetrapolar fungus with clamp connections, such as Schizophyllum commune (Fr.) Fr., heterokaryosis is a prerequisite for clamp formation, and the mechanism is regulated by the incompatibility factors of the heterokaryon. In particular, different $A$ incompatibility factors are necessary for hook cell formation, and different B factors for completion of the clamp (Koltin et al. 1972). Less is known about the genetic mechanisms determining clamp formation in bipolar fungi, though here too the clamp connections, with a few exceptions, are associated with heterokaryosis. In $P$. gigantea the association of clamp connections with heterokaryosis seems to be only partial: some clamp connections appear to be always present in heterokaryons and in most homokaryons, while some homokaryons seem to lack them completely.

Boidin and Lanquetin (1984b) have recently reviewed the current knowledge of the sexuality of the non-poroid Aphyllophorales. Approximately 420 species are listed. Some 32 species have been shown to be primarily homothallic (a few additional species with uninucleate cells are haploid-parthenogenetic). The number of species with "presumed homothallism" is close to 65. Approximately half of them are clampless, or species with infrequent simple clamps. The rest, about 30 species belonging to 13 or 14 genera, are similar to $P$. gigantea in having holocoenocytic nuclear behaviour and multiple clamps. Some of these species may prove to be heterothallic after they have been studied in more detail.

Eriksson et al. (1981) include two European species in the genus Phlebiopsis: $P$. gigantea and $P$. roumeguerii (Bres.) Jülich \& Stalpers. The latter grows on deciduous wood and is southern in distribution. Its sexuality has not been completely solved (Boidin \& Lanquetin 1984b). The clamp connections in this species are rare and simple.

The closest relatives of the genus Phlebiopsis appear to be members of the genera Meruliopsis (Stalpers 1978) and Phanerochaete (Eriksson et al. 1981). Holocoenocytic nuclear behaviour is also characteristic of these genera, and the species are assumed to be homothallic (Boidin \& Lanquetin 1984b). Most species have multiple clamp connections. Primary homothallism has recently been shown in one clampless species of Phanerochaete (Alic \& Gold 1985).

Holocoenocytic nuclear behaviour is also common in the genus Stereum. Boidin and Lanquetin (1984b) list 24 species belonging to this genus; at least half of them are holocoenocytic and were earlier suspected to be homothallic. More recently, however, Rayner and his colleagues investigated the mating behaviour of five Stereum species. Only two of them, S. sanguinolentum (Alb \& Schw.: Fr.) Fr. and S. ochraceoflavum (Schw.) Ellis (= S. rameale (Pers.: Fr.) Burt.), proved homothallic, while the mating system of $S$. gausapatum (Fr.) Fr., S. hirsutum (Willd.: Fr.) S.F. Gray, and S. rugosum (Pers.: Fr.) Fr. turned out to be bipolar, in spite of clamped primary mycelia. The distinction between homo- and heterokaryotic mycelia in these species can be made on the basis of the mycelial morphology (Coates et al. 1981, Boddy \& Rayner 1982, Rayner \& Turton 1982).

Although the external appearance of homo- and heterokaryotic mycelia is also somewhat different in $P$. gigantea, a more useful indicator of heterokaryosis in this species is the ready fruiting of heterokaryotic cultures. However, the occasional occurrence of homokaryotic fruiting makes even this indicator ambiguous (unless the monosporous progeny is analysed; Fig. 3).

Homokaryotic fruiting has been observed in the pure cultures of many heterothallic basidiomycetes (Stahl \& Esser 1976), but in these species it seldom seems to occur in nature, or at least is difficult to observe. It is true, on the other hand, that closely related hetero- and homothallic groups have been found in nature (Hallenberg 1984). Homothallic strains and species have probably arisen from heterothallic species (Ullrich \& Raper 1975). When changing from heterothallism to homothallism, a fungus loses part of its capacity for genetic variation and ecological adaptation, and the final result of this development may be an evolutionary deadlock (Stamberg \& Koltin 1981). However, homothallism offers clear advantages, too. The simple establishment of a new fertile individual from one spore, or from several identical 
spores at the same time, may be an important advantage in competition for exposed substrata, such as freshly cut tree stumps.

In comparison, the establishment of a heterothallic fungal individual requires the penetration of two compatible homokaryons into the substrate. Moreover, they have to find each other and mate. Sexual compatibility is usually not a problem owing to the multiallelic mating factors typical of basidiomycetes. The delay of the heterokaryon in resource capture is partly compensated by the exploitation of the territories of both homokaryons in the substrate. The transfer of nuclei from a heterokaryon to a contiguous homokaryon (Buller phenomenon) produces new heterokaryons. It is unclear whether this exchange of nuclei leads to any physiological cooperation between the donor and receiver mycelium, both of which have an identical nucleus forming one member of the heterokaryotic nuclear pair (Rayner et al. 1984).

Heterothallic $P$. gigantea is a successful colonizer of dead coniferous wood, especially pine. Together with homothallic Stereum sanguinolentum and members of the Sistotrema brinkmannii (Bres.) Erikss. complex, which may also be homothallic (Hallenberg 1984), it belongs to the typical basidiomycetes found in fresh pine stumps cut during the warm season in Finland. The spore infection of these species is very effective, and one year after felling several individuals of $P$. gigantea can usually be found in a single pine stump. These individuals are generally heterokaryotic (Korhonen, unpubl.).

There may be some selectional pressure towards homothallism in $P$. gigantea as well. The relatively frequent homokaryotic fruiting in pure culture might be an indication of such a developmental trend. The capacity for homokaryotic fruiting as such, however, cannot suffice for successful abandonment of sexuality, because the performance of the homokaryons generally appears to be markedly poorer than that of heterokaryons, especially as regards their longevity (Kropp et al. 1987).

When $P$. gigantea is used for the control of $H$. annosum, the freshly cut surface of pine stumps is treated with an oidial suspension. If the oidia originate from one heterokaryotic mycelium, the applied suspension contains both hetero- and homokaryotic propagules. In the stump, heterokaryotic oidia germinate to form heterokaryons which are identical with the original mycelium. Homokaryotic oidia germinate to form two types of homokaryons. These are compatible with each other and mate occasionally, producing a heterokaryon that is also identical with the original one. When the genetically identical heterokaryons meet, they fuse into one mycelial unit. The result is a genetically homogeneous hetero- karyotic mycelium in the stump.

The situation would be different if basidiospores from a heterokaryon were used in the treatment. Homokaryotic basidiospores germinate to form genetically different homokaryons. Matings between them would produce a mosaic of genetically different heterokaryons in the same stump. These mycelia do not fuse but compete with each other. Although experimental evidence is lacking in the case of $P$. gigantea, the basidiospore treatment might not be as effective as oidial treatment owing to this mutual competition (Årsvoll 1976, Rayner et al. 1984). A comparable situation would arise if a mixture of oidia from several fungal individuals was used in the treatment.

If $P$. gigantea is to be used on a large scale to control $H$. annosum in stumps, it might be of interest to try to breed more effective strains. The solution of the sexuality of $P$. gigantea opens up new possibilities for such work.

Acknowledgement: We thank Dr. Keith N. Egger for useful discussions.

\section{References}

Alic, M. \& Gold, M.H. 1985: Genetic recombination in the lignin-degrading basidiomycete Phanerochaete chrysosporium. - Appl. Environm. Microbiol. 50: 27-30.

Årsvoll, K. 1976: Mutual antagonism between isolates of Typhula ishikariensis and Typhula incarnata. - Meld. Norges Landbrukshøgskole 55(19): 1-6.

Biggs, R. 1938: Cultural studies in the Thelephoraceae and related fungi. - Mycologia 30: 64-78.

Boddy, L. \& Rayner, A.D.M. 1982: Population structure, inter-mycelial interactions and infection biology of Stereum gausapatum. - Trans. British Mycol. Soc. 78: $337-351$

Boidin, J. 1950: Sur le comportement nucleaire du carpophore et du mycelium en culture chez Peniophora Cooke. - Comptes Rendus Acad. Sci., Paris, 230: 2328-2330.

Boidin, J. \& Lanquetin, P. 1984a: Répertoire des données utiles pour effectuer les tests d'intercompatibilité chez les Basidiomycètes I. Introduction. - Cryptogamie, Mycologie 5: 33-45.

- 1984b: Indices of useful informations for intercompatibility tests in Basidiomycetes 3. Non poroid Aphyllophorales. Cryptogamie, Mycologie 5: 193-245.

Coates, D., Rayner, A.D.M. \& Todd, N.K. 1981: Mating behaviour, mycelial antagonism and the establishment of individuals in Stereum hirsutum. - Trans. British Mycol. Soc. 76:41-51.

Eriksson, J., Hjortstam, K. \& Ryvarden, L. 1981: The Corticiaceae of North Europe 6: Phlebia - Sarcodontia. - Pp. 1049-1276. Oslo.

Gilbertson, R.L. \& Ryvarden, L. 1986: North American Polypores 1. Abortiporus - Lindtneria. - 433 pp. Oslo.

Hallenberg, N. 1984: A taxonomic analysis of the Sisto- 
trema brinkmannii complex (Corticiaceae, Basidiomycetes). - Mycotaxon 21: 394-411.

Käärik, A. \& Rennerfelt, E. 1957: Investigations on the fungal flora of spruce and pine stumps. - Meddel. Stat. Skogsforskningsinstitut 47(7): 1-88.

Kemp, R.F.O. 1980: A heterothallic species of Coprinus related to $\mathrm{C}$. sterquilinus with clamp-connexions on homokaryotic and dikaryotic mycelia. - Trans. British Mycol. Soc. 74: 418-421.

Koltin, Y., Stamberg, J. \& Lemke, P.A. 1972: Genetic structure and evolution of the incompatibility factors in higher fungi. - Bact. Rev. 36: 156-171.

Korhonen, K. \& Hintikka, V. 1981: Simple isolation and inoculation methods for fungal pure cultures. Karstenia 20: 19-22.

Kropp, B.R., McAfee, B.J. \& Fortin, J.A. 1987: Variable loss of ectomycorrhizal ability in monokaryotic and dikaryotic cultures of Laccaria bicolor. - Canad. J. Bot. 65: 500-504.

Rayner, A.D.M., Coates, D., Ainsworth, A.M., Adams, T.J.H., Williams, E.N.D. \& Todd, N.K. 1984: The biological consequences of the individualistic mycelium. - In: Jennings, D.H. and Rayner, A.D.M. (eds.), The ecology and physiology of the fungal mycelium. British Mycol. Soc. Symp. 8: 509-540.

Rayner, A.D.M. \& Turton, M.N. 1982: Mycelial interactions and population structure in the genus Stereum: S. rugosum, S. sanguinolentum and S. rameale. - Trans. British Mycol. Soc. 78: 483-493.

Rishbeth, J. 1979: Modern aspects of biological control of Fomes and Armillaria. - Eur. J. Forest Path. 9: 331340.

Schulz-Weddingen, I.H. 1985: Coprinus verticillatus, a new agaric with multiple clamps. - Mycologia 77: 154158.

Stahl, U. \& Esser, K. 1976: Genetics of fruit body production in higher Basidiomycetes 1. Monokaryotic fruiting and its correlation with dikaryotic fruiting in Polyporus ciliatus. - Molec. Gen. Genet. 148: 183-197.

Stalpers, J.A. 1978: Identification of wood-inhabiting fungi in pure culture. - Studies in Mycology 16: 1-248. Baarn.

Stamberg, J. \& Koltin, Y. 1981: The genetics of mating systems: fungal strategies for survival. - In: Wicklow, D.T. \& Carroll, G.C. (eds.), The fungal community, its organization and role in the ecosystem: 157-170. New York \& Basel.

Ullrich, R.C. \& Raper, J.R. 1975: Primary homothallism - relation to heterothallism in the regulation of sexual morphogenesis in Sistotrema. - Genetics 80: $311-321$.

Accepted for publication on 17 September 1987 